\title{
Too much or too little
} medicine? Overdiagnosis, underdiagnosis, overtreatment and undertreatment in respiratory diseases

Cite as: Dobler CC. Too much or too little medicine? Overdiagnosis, underdiagnosis, overtreatment and undertreatment in respiratory diseases. Breathe 2019; 15: 2-3.

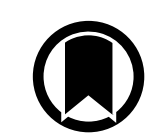

CrossMark
At a time when technical possibilities for medical investigations are plentiful and ever expanding, there is growing awareness that more is not always better and that "too much medicine" may be harmful. In recent years, the global Choosing Wisely educational campaign has aimed to bring attention to unnecessary healthcare. Unnecessary healthcare includes overtesting, overdiagnosis and overtreatment [1]. The March issue of Breathe aims to challenge us to rethink our clinical practice, to reflect on the evidence, and to identify potential cognitive biases that might influence us to provide "too much" or "too little" medicine [2].

This issue contains a number of articles on the topic of over- and underdiagnosis and over- and undertreatment in respiratory diseases. The procon articles on whether asymptomatic patients with moderate-to-severe sleep apnoea should be treated [3, 4] debate a hotly contested topic in sleep medicine and illustrate that evidence is not self-explanatory but needs to be interpreted. Facts are not neutral; they are theory- and value-laden $[5,6]$, and different people may arrive at varying conclusions based on the same evidence. An article that examines the evidence for screening for sleep disordered breathing in pregnancy, challenges us as clinicians to communicate uncertainty in the evidence to patients [7]. An article giving a global perspective of over- and underdiagnosis of COPD highlights the seeming paradox that under- and overdiagnosis of COPD coexist in the same setting [8]. Further, the question of whether "aggressive" (surgical) investigation of solitary pulmonary nodules can be justified [9] and the topic of overdiagnosis of pulmonary embolism [10] are explored. Further articles will be published as online exclusives for the March issue. Keep checking the Breathe website for newly added content.

We continue the Journal club series that was successfully launched in the December 2018 issue of Breathe. I would like to thank Frits Franssen who is the new section editor for the Journal club papers and Cristina Calarasu who is coordinating the commissioning of papers with the early career members of the European Respiratory Society. Topics include: excessive care in the intensive care unit [11]; low-dose CT instead of chest radiography in suspected pneumonia [12]; and procalcitoninguided antibiotic therapy in lower respiratory tract infections [13]. The lung function corner explores the use of the transfer coefficient of the lung for carbon monoxide and the accessible alveolar volume [14].

The thread topics for upcoming Breathe issues are now listed on the website to allow potential contributors to plan well in advance. For 2019 we have planned the following threads: quality of life/living 
well with a chronic respiratory disease (June), oxygen (September) and biomarkers (December).

I hope that you enjoy this edition of Breathe and leave you with the words of Gilbert $\mathrm{H}$. Welch to reflect upon:
"True professionals provide considered advice. And sometimes doing nothing is exactly the right thing to do. The same is true of medicine. Recognize that the doctor who advises no action may be the one who really cares for you."

\section{Affiliations}

\section{Claudia C. Dobler}

Dept of Respiratory Medicine, Liverpool Hospital, and University of New South Wales, Sydney, Australia.

\section{Conflict of interest}

C.C. Dobler has nothing to disclose.

\section{References}

1. Carter SM, Rogers W, Heath I, et al. The challenge of overdiagnosis begins with its definition. BMJ 2015; 350: h869.

2. Dobler CC, Morrow AS, Kamath CC. Clinicians' cognitive biases: a potential barrier to implementation of evidence-based clinical practice. BMJ Evid Based Med 2018; in press [https://doi. org/10.1136/bmjebm-2018-111074].

3. Ryan S. Pro: should asymptomatic patients with moderateto-severe OSA be treated? Breathe 2018; 15: 7-10.

4. Vakulin A, Chai-Coetzer CL, McEvoy RD. Con: should asymptomatic patients with moderate-to-severe OSA be treated? Breathe 2018; 15: 11-14.

5. Kelly MP, Heath I, Howick J, et al. The importance of values in evidence-based medicine. BMC Med Ethics 2015; 16: 69.

6. Wieringa S, Engebretsen E, Heggen K, et al. Rethinking bias and truth in evidence-based health care. J Eval Clin Pract 2018; 24: 930-938.

7. Perkins A, Einion A. Pregnant pause: should we screen for sleep disordered breathing in pregnancy? Breathe 2018; 15 : 36-44.
8. Ho T, Cusack R, Chaudhary N, et al. Under- and over-diagnosis of COPD: a global perspective. Breathe 2018; 15: 24-35.

9. Elia S, Loprete S, De Stefano A, et al. Does aggressive management of solitary pulmonary nodules pay off? Breathe 2018; 15: 15-23.

10. Dobler CC. Overdiagnosis of pulmonary embolism: definition, causes and implications. Breathe 2018; 15: 46-53.

11. Okenyi E, Donaldson TM, Collins A, et al. Assessing ethical climates in critical care and their impact on patient outcomes. Breathe 2018; 15: 84-87.

12. Hoesein FM. Low-dose computed tomography instead of radiography in suspected pneumonia. Breathe 2018; 15 : 81-83.

13. Mathioudakis AG, Vestbo J. Was the implementation strategy of the ProACT trial adequately proactive? Breathe 2018; 15: 77-80.

14. Neder JA, Marillier M, Bernard A-C, et al. Transfer coefficient of the lung for carbon monoxide and the accessible alveolar volume: clinically useful if used wisely. Breathe 2018; 15: 69-76 